\title{
Pharmacology in the age of the Holobiont
}

Povilas Norvaisas ${ }^{1}$ and Filipe Cabreiro ${ }^{1,2}$

1. Institute of Structural and Molecular Biology, University College London and Birkbeck, London WC1E 6BT, UK

2. Corresponding author. Email: f.cabreiro@ucl.ac.uk

\section{Highlights}

- Pharmacomicrobiomics - new frontier in pharmacology

- Holobiont - the physiological unit of host and microbes as a drug target

- Drugs and drug targets are re-defined in the context of the holobiont

- New holistic approach in drug research using holobiont animal models

- Exciting new prospects for microbiota-based personalised medicine

\section{Glossary:}

Holobiont - Supra-organism of host and its microbes.

Pharmacogenomics - Field of research which combines pharmacology and genomics to study how the genetic make-up of an individual modulates its response to therapeutic drugs [1].

Pharmacometabolomics - Field of research which combines pharmacology and metabolomics to study how the metabolome shaped by environment and genetics influence the response of an individual to therapeutic drugs [2].

Xenobiotics - An exogenously produced chemical compound found inside an organism. This broad term encompasses therapeutic drugs and dietary metabolites.

\begin{abstract}
Despite the widely acknowledged fact that the microbiota regulates many aspects of human health, the dynamics and factors that govern these interactions remain mostly unknown. Pharmacomicrobiomics is a new research frontier in pharmacology that studies the interaction between drugs and the microbiota. This discipline, by including the microbiota as a key regulator of host health, calls for a redefinition of what constitutes a drug target and ultimately what is a drug or drug therapy. This is supported by recent evidence showing that host physiology can no longer be studied in separation from its microbial ecology and the environmental factors that shape it, as the combination of these elements forms the physiological unit of study - the holobiont. Here we discuss both the novel challenges and untapped opportunities that this new framework creates. On one hand, a more complete understanding of the physiology of the host imposes the development/adaptation of new animal models to address these interactions. In particular, we focus on the advantages and disadvantages of $C$. elegans as a host organism. On the other hand - a complete understanding of the effects of the microbiota and xenobiotics (e.g. drugs and dietary metabolites) on host health opens new prospects for personalized therapy.
\end{abstract}




\section{Introduction}

Pharmacology has come a long way since the inception of "magic bullet" drugs at the beginning of the 20th century. Intelligent drug design still holds the promise of devising new compounds that specifically affect a disease target yet are harmless to the functioning of the entire organism. The lack of success in achieving such gold standards, associated with high failure of new drugs in clinical trials has led to an increasing interest for drug repurposing [3,4]. Developments in molecular and cellular biology have revealed a number of new drug targets and factors that may affect efficacy and/or side-effects. As a consequence, new branches of pharmacology emerged, like pharmacogenomics and pharmacometabolomics, which aim at understanding how the genetics and the metabolism of an individual can modulate drug efficacy.

Despite great advances in pharmacology, one factor often disregarded in developing personalised medicine is the role of the gut microbiota in the modulation of drug effects. The microbes inhabiting the gut of an organism have a dramatic role in regulating host health [5] and are the target of an unexpected high number of drugs originally designed at targeting host processes [6]. As a consequence, the recent revolution in microbiome research established a new frontier in pharmacology. Pharmacomicrobiomics aims at tackling the interplay between drugs, microbiota ecology, environmental pressures and host genotype [7-9]. The full scope of these interactions may only be understood using holistic approaches, thus requiring a shift in our understanding of the human body. Together with their microbiota, animals form the holobiont [10] - a single physiological entity with a combined metagenome, and metabolism that is shaped by its environment [11,12] (Figure 1a). Therefore, redefining the holobiont as a target of drug therapy rather than the host only, may also call for a re-evaluation of what therapy is, to include compounds that directly or indirectly affect drug efficacy through an indirect impact on the microbiota.

The holobiont exists in a constant flux of dietary metabolites and drugs, which are taken from the environment and circulated between the host and its microbes. In terms of their biological effect, these compounds can be foodstuff, inert, regulatory or toxic molecules. Often there is a shared chemical space between the host and the microbiota co-metabolism. In this context, microbes and host share identical or similar metabolic pathways allowing them to exchange and utilize metabolites indiscriminately within the holobiont. In other cases, microbes and host complement each other metabolically - where the products of the metabolic machinery of one organism can be utilized by the other (Figure 1b). For example, microbes utilize dietary fibre as a nutrient source [13,14], which is mostly inert matter for the human host, and the resulting short-chain fatty acids positively regulate host physiology. However, these concepts are relative and ultimately depend on the specific microbes and the host cell type [15,16]. Ultimately, the phenotype of the holobiont is shaped by the metabonome [17] shared between the microbiota and the host that operates in a complex chemical feedback loop (Figure 1c). Therefore, transient environmental factors like diet and drugs may alter the function of the microbiota leading to the production of metabolites that can act as adjuvants or impair drug action on host metabolism [7,18] or alternatively, may impact the direct transformative role of the microbiota in chemically modifying drugs [19]. In such scenarios causality in drug action may easily be lost leading to unanticipated and poorly understood mechanistic effects. This further highlights the increasing need to adopt such an integrative view of physiology and pharmacology for drug research and development.

Altogether, the recent developments in pharmacology stress the uniqueness of each 
patient as a result of its genome, microbiome and intricate relationship with the environment. Such complexity underscores the challenges ahead for precise personalized medicine but also reveals a myriad of untapped opportunities for therapy [20]. So, what is needed to bring pharmacology into the age of microbiome? How can we use animal models to feasibly, accurately and predictively tackle the ever-increasing complexity of drug action and ultimately draw insights which will lead to the improvement of therapies? In this short review, we highlight some of the key ideas at the intersection of microbiome and pharmacology research and provide hints for potential future trends in these fields.

\section{Targeting the structure of the microbiome for health}

The human microbiota is formed of trillions of bacterial cells, viruses and fungi that inhabit the various anatomical regions of the human body [21] and there is a general consensus that it plays a crucial role in host health [11]. The in-depth analysis of the structure and the composition of our microbiota was enabled by the shift to a cultureindependent approach using $16 \mathrm{~S}$ rRNA and shotgun metagenomic sequencing [22]. Major population studies have revealed that the microbiome is remarkably stable in healthy adults [23] and vastly rich in metabolic functions [24,25]. It contains 100x more genes than the human genome, with $50 \%$ of its content not fully annotated and likely to contribute greatly to the extended metabolic capacity of the holobiont [11,12]. Each persons' microbiome is unique and exhibits wide inter-personal and intra-personal variation, with distinct bacterial communities inhabiting different anatomical regions [21]. Given the complexity of this ecological niche and its quickly adaptable nature, it is not only difficult to define the core composition of a healthy microbiota but also to fully capture and define the mechanisms that govern the transitions between healthy and unhealthy states [26]. Additionally, since most human microbiome studies are retrospective and our control of the variables is governed by ethical or technical limitations, unknown factors may drive structural changes in microbiome communities and thus unknowingly define the holobiont phenotype. This was illustrated by the driving effects of the antidiabetic drug metformin on the structure of the microbiota in a human type-2-diabetes (T2D) study [27]. Two gut metagenomics studies of T2D patients that did not take drug treatment as a key confounding variable yielded conflicting conclusions regarding the nature of the disease-associated gut microbial dysbiosis [28,29]. Disentangling drug effects allowed for the identification of a unified microbiome signature induced by $\mathrm{T} 2 \mathrm{D}$ and revealed a positive impact of metformin on the microbiota contributing partly to its therapeutic effects [27].

Therefore, the lack of a complete mechanistic understanding of such microbiome shifts is troubling, because the adaptable nature of the microbiome also makes the holobiont phenotype a moving target for therapy. One potential solution is the stratification of microbial communities into functional states called enterotypes [30], as major indicators of structural trends. Such strategies may ultimately become useful in the clinical setting, providing an opportunity for the microbiome's systematic investigation, classification and potential targeted alteration.

\section{Harnessing the power of the microbiome - Drugs}

The ecological niche of the human microbiota can be shaped and perturbed by multiple factors [11]. Firstly, it is defined by the physical properties of the anatomical location [21], the genotype [31] and the age of the host [32,33]. Then, multiple transient environmental factors, over the lifetime of the host, alter or perturb its microbiota ecology, 
namely lifestyle [34], infections [35], hygiene [36], diet [15,37-39] and exposure to xenobiotics $[7,16,40]$ (Figure 1a). Research suggests that environmental factors are the major drivers in shaping gut microbial communities, overruling host genotype [37,41] $[25,42]$. Remarkably, in vitro assays show that $24 \%$ of over a 1000 of host-targeted drugs impaired the growth of at least 1 out of 40 representative human gut bacterial strains [6] supporting a key role for drugs modulating the microbiota. The antidiabetic drug metformin, also identified in this study, portraits part of the complexity involved in holobiont-drug interactions. Studies show that the drug has distinct effects on both host and microbial cells but the combined interactions of drug-host plus drug-microbe determine the holobiont phenotype. In fact, metformin was shown to induce important structural $[27,43]$, transcriptional [43] and metabolic changes [44] in the microbiota, but also in the metabolism of the host [1], ultimately contributing to its efficacy in the regulation of host glucose homeostasis [43] and lifespan [44]. Additionally, the microbiota can indirectly modulate drug effects by producing compounds that can compete directly with drugs (e.g. acetaminophen and p-cresol) for their modification by enzymes of the host [7].

Microbes can also directly alter drug pharmacokinetics by activating, reactivating and inactivating drugs. Currently, around 60 drugs are known to undergo several types of chemical modification by the gut microbiota [7,9]. This forms an important component of the first-pass drug metabolism together with the intestinal and hepatic enzymes of the host. Prodrugs, like protonsil, can be activated by microbial azoreductases cleaving their azo bond - a feature that was accounted for during drug design. Conversely and unexpectedly, up to $50 \%$ of cardiac glycoside digoxin can be rendered inactive through biochemical reduction by Eggerthela lenta [7]. Two additional types of co-metabolism in the context of drugs are elegantly illustrated by the anticancer drugs irinotecan [45] and fluoropyrimidines [46]. The anticancer agent irinotecan is inactivated by hepatic glucuronidation and then reactivated by bacterial b-glucuronidases after biliary secretion. This also demonstrates that oral intake is often not a prerequisite for drug-microbe interactions due to biliary and epithelial excretion. Fluoropyrimidine pro-drug efficacy, on the other hand, depends on the relative contribution of the metabolic ribonucleotide pathways of the host and its microbes to biochemically activate these compounds and ultimately target host cellular division [46,47]. Further, metabolites and molecular cues from E. coli and F. nucleatum, respectively, modulate the activation of cellular autophagy and thus the effect of fluoropyrimidines $[46,48]$ on cancer progression.

Altogether, these studies illustrate two important principles. One, that drugs can be used to selectively edit the microbiota by suppressing or increasing the abundance of specific bacterial strains involved in regulating host phenotypes (e.g metformin [27,43]). Two, dietary cues or drug-drug combinations could be used in tandem as tools for modulating the bi-directional metabolic/signalling communication that exists between host and microbe in the context of drug efficacy. Overall, given the responsive nature and the unique role of specific microbes in maintaining different aspects of health [49], the microbiota provides exciting prospects to be explored either as a therapy itself or as a target of drugs $[44,50]$ for health benefits.

\section{Harnessing the power of the microbiome - Diet}

Given the microbiota's sensitivity to a diverse range of xenobiotics apart from hosttargeted drugs and antibiotics, personalised nutrition has emerged as a new therapeutic paradigm. Diet is therefore being viewed as an instrument to shape the microbiota and in 
turn, the holobiont phenotype $[8,18,38,41]$. Such modification may involve the use of prebiotics (e.g dietary fiber, inuli type-fructans), which shape the microbiota by promoting growth of specific bacterial phyla [15] and provide general benefits to the host [13,14,51]. However, little is known about the mechanisms by which nutrients and/or metabolites from our diets influence the holobiont. Human diets are naturally complex, composed of an undefined mishmash of macro and micronutrients. Thus, understanding the effect of a single or a combination of nutrients/metabolites on the holobiont phenotype is an essential but daunting task. The metabolism of L-carnitine, an important dietary component of red meat, is an example of complementary metabolism for dietary cues between the microbiota and host, with implications for the health of the holobiont. Bacteria convert L-carnitine to trimethylamine which is further metabolised by the liver of the host to trimethylamine $\mathrm{N}$ oxide, a product that promotes atherosclerosis [52]. Another example is provided by the metabolism of tryptophan, an aromatic amino acid. Tryptophan, can either be converted by gut bacteria to produce indoles that improve healthspan [53] or alternatively, the uremic toxin indoxyl sulphate which can cause chronic kidney disease [54] (Figure 1b). Therefore, to better understand the effects of nutrition and define dietary approaches as therapy, we first need to develop comprehensive and integrative methods that allow the causal investigation of the complex interactions between host, microbes and nutrition in the regulation of the phenotype and health of the holobiont.

\section{Experimental holobiont models}

Microbiota research is moving away from descriptive correlative studies to in-depth mechanistic analysis of both its function and therapeutic implications. The complexity of the holobiont is intimidating due to a vast number of variables that shape its phenotype. Some of these challenges can be met by experimental animal models, but they need to fulfil a number of requirements to provide new insights on the biology of the holobiont $[12,55]$ (Figure 2a). Bacterial community ecology remains challenging to investigate, model and engineer [56,57]. Microbial ecosystems can be studied through phenotype screening of cultivable microbes [58]. This allows the characterization of the microbes in this community based on general physiological properties, sensitivity to drugs [6] and nutritional preferences [59]. Additionally, it provides a platform for the detailed modelling of shared metabolism between bacterial members [60]. Semi-artificial cellular platforms created by gut-on-chip organoid models [61] may be used to reveal the biological principles in the interaction between complex eukaryotic cellular environments and prokaryotic ones. The simplest whole-organism model with a microbiota of an intermediate complexity is $C$. elegans [62,63]. Easy to handle, cost-effective and scalable, it allows the systematic highthroughput investigation of the effects of the microbiota on a host's phenotype [64]. Drosophila is unique in its defined holidic nutritional media [65] and a simple native microbiota comprising of approximately 20 bacterial species, which allow the study of nutrition-microbiota-host interactions in diverse evolutionarily conserved host traits [12,6668]. Zebrafish and Nothobranchius Furzeri are emerging as powerful models to study microbiota effects in vertebrates. For example, the transparency of zebrafish enables the live and real-time investigation of gut colonisation dynamics using fluorescently-labelled bacterial strains [69]. N. Furzeri has become instrumental for the study of the role of the microbiota in organismal ageing due to its relatively short lifespan for a vertebrate animal [70]. The common mammalian model of choice are mice [37,71], whose microbiota has been standardised [72] and can also be humanised [73], even though it lacks the scalability 
offered by lower animal and non-animal models. However, the closest to humans but rarely used model is the pig, which can also receive and maintain human microbiota [74]. Overall, all models provide unique benefits but also distinctive drawbacks and the researcher's choice should ultimately depend on the scope of the study and the nature of the problem that is being investigated.

C. elegans is the simplest complete holobiont model organism, providing proxy readouts for most aspects of host-microbiota interactions. It is uniquely scalable and can feasibly address the impact of environmental cues (e.g. drug, nutrition), host genetics and microbial genetics in different aspects of holobiont physiology (e.g development, reproduction, metabolism, ageing) (Figure 2b). C. elegans has a short generation time of 3 days and a lifespan of 3 weeks with isogenic, transgenic and gnotobiotic animals easily maintained. It is arguably the best genetic multicellular model with powerful forward and reverse genetics screen tools, in addition to transgenesis and CRISPr/Cas9 gene editing tools for the identification of genes and their function in the regulation of holobiont phenotypes. Its native microbiome is intermediately complex [62,63], but worms can also grow in lab conditions on a single or a community of bacterial strains which act as both nutritional source and gut commensals [64]. Unfortunately, worms are normally cultured at ambient levels of oxygen, which imposes a restriction on the study of microbes that are mainly aerobic. However, it remains to be investigated whether the worm gut is microaerophilic or anaerobic, with the potential capacity to maintain strict or facultative anaerobes. Given the critical relationship between gut microbes and host immunity, the lack of an adaptive immune system is a drawback of this holobiont system, which can impose limits on the translatability of findings to humans.

The greatest advantage of $C$. elegans in comparison to other models is its highthroughput screening capacity, not lagging far behind classical high-throughput microbiology approaches [16,46,75]. For example, bacterial knockout E. coli libraries [76] can be used together with wildtype worms $[77,78]$ or possibly in tandem with RNAi libraries [79], or mutation genetic libraries [80] for the study of the interaction between microbial genetics and host genetics. The worm can also be used to assess the influence of bacterial genetics in regulating drug efficacy on host physiology, such as the role of anticancer drugs $[46,47,81]$ or metabolic drugs such as metformin [44]. In addition, GFP transcriptional reporter E. coli libraries [82] could be used to study the influence of host genetics on the regulation of bacterial gene expression within a community of microbes during the gut colonization - all enabled by the transparency of host's body. With more widespread adaptation, the simplicity of this model will yield unique advantages in resolving complex problems. It may, for example, enable early high-throughput in vivo testing in drug development pipelines and provide an excellent platform for the discovery of bacterial genes and/or processes regulating evolutionary conserved mechanisms in the holobiont.

\section{Conclusions}

The widespread role of the microbiota in regulating the health and wellbeing of the holobiont provides exciting avenues for therapy but our current understanding of the complex interactions between host, microbiota and its environment is still in its infancy. Therefore, research focusing on the microbiota has the potential to drastically change pharmacology and our approach to human therapies. As the most recent iteration of personalised medicine, pharmacomicrobiomics highlights the necessity to investigate different metabolic parameters of the microbiota in order to avoid spurious drug 
interactions and allow the development of more robust therapies. Its implications extend far beyond prevention of undesired interactions, with the possible targeted use of xenobiotics to alter microbiota for host health benefits. However, the ability to temper with the microbiota for therapeutic purposes requires a more systematic and mechanistic investigation of the holobiont, to define the functional states of the microbiota, the factors that shape it and their biological effects on the host. Such approaches may not be able to rely on the known sets of scaffolds and drug-likeness principles of compounds aimed at the host. New guidelines may result from pharmacomicrobiomic research as we build on the solid mechanistic understanding of basic processes and extrapolate them to more complex settings. Scalable holobiont models, in particular C. elegans, already embody such philosophy and allow the detailed and controlled investigation of multiple factors that shape the physiology of the holobiont. Ultimately, discoveries made in animal models should lead to therapeutic predictions using metabolic models [57] (Figure 2c). For example, important progress has already been made in establishing the necessary computational tools [83] and reconstructing metabolic models of bacterial strains from the human microbiota [84]. There is also a community effort to develop the first metabolic model of a multicellular organism - C. elegans, which will incorporate the metabolic contribution of gut commensals [85]. In the future, such approaches might provide valuable insights when transitioning from animal testing to human trials. These are the very first steps in the field of pharmacomicrobiomics that will likely introduce new therapies, but more than that, it will without a doubt bring a more complete understanding of our health.

\section{Figure captions:}

Figure 1: (a) Holobiont - a holistic framework in pharmacology which combines host, microbiota and environmental interactions. Traditional pharmacology concentrates primarily on the drug effects on host physiology with respect to the variation in host genome (pharmacogenomics) and metabolome (pharmacometabolomics). However, host development, health and homeostasis intricately depend on microbial ecology, which itself can become a target of therapy (pharmacomicrobiomics). This reveals both exciting opportunities and daunting challenges, as the microbiota can be easily moulded by multiple environmental factors. (b) The holobiont exists in a flux of xenobiotics (e.g. dietary metabolites and drugs) which may have differing effects on host and bacterial cells. Bacteria may also transform these compounds (green arrows) thus contributing to their indirect effects. (c) The Holobiont results from the combination of the metagenome and metabonome shared by the host and its microbiota and shaped by a complex feedback loop resulting from their interaction.

Figure 2: (a) General comparison of experimental holobiont animal models and humans in terms of host, microbiota and environmental properties. (b) C. elegans - scalable holobiont model, which can be used in high-throughput screens to unravel the complexity of host, microbiota and environment interactions. (c) Findings in experimental setting may be integrated using $C$. elegans and E. coli metabolic model and further extrapolated to humans.

\section{Acknowledgements}

F.C. acknowledges funding from the Wellcome Trust/Royal Society (Sir Henry Dale 
Fellowship-102532/Z/12/Z) and P.N. from the Wellcome Trust. We would like to thank Dr. Kevin Bryson for critical reading of the manuscript. We apologize for the exclusion of some of the research findings and less commonly used holobiont models due to the limited scope of this review.

\section{Recommended reading ( ${ }^{*}$ of special interest, ${ }^{* *}$ of outstanding interest):}

- ** Scott 2017 \& Garcia-gonzalez 2017 - First studies of the mechanisms by which commensal microbes alter the efficacy of anticancer fluoropyrimidine drugs using a C. elegans holobiont model. Development of 3-way host-microbe-drugs highthroughput screens.

- $\quad$ ** Zeevi 2015 - In this study patient glycemic response to diet was successfully predicted using their microbiota composition and other health metrics. These predictions were then used to correspondingly change patient diet. This highlights the use of personalized nutrition as a therapeutic strategy.

- ** Leulier 2017 - This paper highlights the need for an integrative physiology framework which incorporates both microbiota and diet as important factors shaping the host phenotype.

- ** Maier 2018 - In this study, 40 gut microbiota representative bacterial strains were challenged with over 1000 host-targeted drugs. This study highlights the extensive impact drugs can have on the microbiota which can be responsible for drug associated side-effects and/or health benefits.

- * Cabreiro 2013 - First study that shows that the antidiabetic drug metformin induces changes both in microbial metabolism and the host, which ultimately contribute to the phenotype of the host.

- * Stappenbeck 2016 - This paper highlights the challenges of performing microbiota research using traditional animal models.

- * Spanogiannopoulos 2016 - This paper presents a detailed overview of drug/xenobiotic-microbiota interactions.

\section{Bibliography}

1. Relling MV, Evans WE: Pharmacogenomics in the clinic. Nature 2015, 526:343-350.

2. Everett JR: From Metabonomics to Pharmacometabonomics: The Role of Metabolic Profiling in Personalized Medicine. Frontiers in Pharmacology 2016, 7.

3. Pryor R, Cabreiro F: Repurposing metformin: an old drug with new tricks in its binding pockets. Biochemical Journal 2015, 471:307-322.

4. Slack C, Alic N, Foley A, Cabecinha M, Hoddinott MP, Partridge L: The Ras-Erk-ETSSignaling Pathway Is a Drug Target for Longevity. Cell 2015, 162:72-83.

5. Gilbert JA, Blaser MJ, Caporaso JG, Jansson JK, Lynch SV, Knight R: Current understanding of the human microbiome. Nature Medicine 2018, 24:392-400.

6. Maier L, Pruteanu M, Kuhn M, Zeller G, Telzerow A, Anderson EE, Brochado AR, Fernandez $\mathrm{KC}$, Dose $\mathrm{H}$, Mori $\mathrm{H}$, et al.: Extensive impact of non-antibiotic drugs on human gut bacteria. Nature 2018, 555:623-628. 
7. Spanogiannopoulos P, Bess EN, Carmody RN, Turnbaugh PJ: The microbial pharmacists within us: a metagenomic view of xenobiotic metabolism. Nature Reviews Microbiology 2016, 14:273-287.

8. Kuntz TM, Gilbert JA: Introducing the Microbiome into Precision Medicine. Trends in Pharmacological Sciences 2017, 38:81-91.

9. Wilson ID, Nicholson JK: Gut microbiome interactions with drug metabolism, efficacy, and toxicity. Translational Research 2017, 179:204-222.

10. Rosenberg E, Zilber-Rosenberg I: Microbes Drive Evolution of Animals and Plants: the Hologenome Concept. MBio 2016, 7:e01395.

11. Kundu P, Blacher E, Elinav E, Pettersson S: Our Gut Microbiome: The Evolving Inner Self. Cell 2017, 171:1481-1493.

12. Leulier F, MacNeil LT, Lee Wj, Rawls JF, Cani PD, Schwarzer M, Zhao L, Simpson SJ: Integrative Physiology: At the Crossroads of Nutrition, Microbiota, Animal Physiology, and Human Health. Cell Metabolism 2017, 25:522-534.

13. Schroeder BO, Birchenough GMH, Ståhlman M, Arike L, Johansson MEV, Hansson GC, Bäckhed F: Bifidobacteria or Fiber Protects against Diet-Induced MicrobiotaMediated Colonic Mucus Deterioration. Cell Host and Microbe 2017, 23:27-40.

14. Catry E, Bindels LB, Tailleux A, Lestavel S, Neyrinck AM, Goossens J-F, Lobysheva I, Plovier H, Essaghir A, Demoulin J-B, et al.: Targeting the gut microbiota with inulintype fructans: preclinical demonstration of a novel approach in the management of endothelial dysfunction. Gut 2017, 67:271-283.

15. Sonnenburg JL, Bäckhed F: Diet-microbiota interactions as moderators of human metabolism. Nature 2016, 535:56-64.

16. Maier L, Typas A: Systematically investigating the impact of medication on the gut microbiome. Current Opinion in Microbiology 2017, 39:128-135.

17. Everett JR: Pharmacometabonomics in Humans: A new Tool for Personalised Medicine. 2015, 16:1-28.

18. Suez J, Elinav E: The path towards microbiome-based metabolite treatment. Nature Microbiology 2017, 2:1-5.

19. Klünemann M, Schmid M, Patil KR: Computational tools for modeling xenometabolism of the human gut microbiota. Trends in Biotechnology 2014, 32:157-165.

20. Zmora N, Zeevi D, Korem T, Segal E, Elinav E: Taking it Personally: Personalized Utilization of the Human Microbiome in Health and Disease. Cell Host and Microbe 2016, 19:12-20.

21. Donaldson GP, Lee SM, Mazmanian SK: Gut biogeography of the bacterial microbiota. Nature Reviews Microbiology 2016, 14:20-32.

22. Quince C, Walker AW, Simpson JT, Loman NJ, Segata N: Shotgun metagenomics, from sampling to analysis. Nature Biotechnology 2017, 35:833-844.

23. Mehta RS, Abu-Ali GS, Drew DA, Lloyd-Price J, Subramanian A, Lochhead P, Joshi AD, Ivey $\mathrm{KL}$, Khalili H, Brown GT, et al.: Stability of the human faecal microbiome in a cohort of adult men. Nature Microbiology 2018, 3:347-355.

24. Lloyd-Price J, Mahurkar A, Rahnavard G, Crabtree J, Orvis J, Hall AB, Brady A, Creasy HH, McCracken C, Giglio MG, et al.: Strains, functions and dynamics in the expanded Human Microbiome Project. Nature 2017, 550:61-66.

25. Falony G, Joossens M, Vieira-Silva S, Wang J, Darzi Y, Faust K, Kurilshikov A, Bonder MJ, Valles-Colomer $M$, Vandeputte $\mathrm{D}$, et al.: Population-level analysis of gut microbiome variation. Science 2016, 352:560-564. 
26. Manor O, Borenstein E: Systematic Characterization and Analysis of the Taxonomic Drivers of Functional Shifts in the Human Microbiome. Cell Host and Microbe 2017, 21:254-267.

27. Forslund K, Hildebrand F, Nielsen T, Falony G, Le Chatelier E, Sunagawa S, Prifti E, VieiraSilva S, Gudmundsdottir V, Krogh Pedersen $\mathrm{H}$, et al.: Disentangling type 2 diabetes and metformin treatment signatures in the human gut microbiota. Nature 2015, 528:262-266.

28. Qin J, Li Y, Cai Z, Li S, Zhu J, Zhang F, Liang S, Zhang W, Guan Y, Shen D, et al.: A metagenome-wide association study of gut microbiota in type 2 diabetes. Nature 2012, 490:55-60.

29. Karlsson FH, Tremaroli V, Nookaew I, Bergström G, Behre CJ, Fagerberg B, Nielsen J, Bäckhed F: Gut metagenome in European women with normal, impaired and diabetic glucose control. Nature 2013, 498:99-103.

30. Costea PI, Hildebrand F, Arumugam M, Bäckhed F, Blaser MJ, Bushman FD, Vos WMd, Ehrlich SD, Fraser CM, Hattori $M$, et al.: Enterotypes in the landscape of gut microbial community composition. Nature Microbiology 2017, 3:8-16.

31. Goodrich JK, Waters JL, Poole AC, Sutter JL, Koren O, Blekhman R, Beaumont M, Van Treuren W, Knight R, Bell JT, et al.: Human genetics shape the gut microbiome. Cell 2014, 159:789-799.

32. Clark RI, Salazar A, Yamada R, Fitz-Gibbon S, Morselli M, Alcaraz J, Rana A, Rera M, Pellegrini M, Ja WW, et al.: Distinct Shifts in Microbiota Composition during Drosophila Aging Impair Intestinal Function and Drive Mortality. Cell Rep 2015, 12:1656-1667.

33. Thevaranjan N, Puchta A, Schulz C, Naidoo A, Szamosi JC, Verschoor CP, Loukov D, Schenck LP, Jury J, Foley KP, et al.: Age-Associated Microbial Dysbiosis Promotes Intestinal Permeability, Systemic Inflammation, and Macrophage Dysfunction. Cell Host \& Microbe 2017, 21:455-466.

34. David LA, Materna AC, Friedman J, Campos-Baptista MI, Blackburn MC, Perrotta A, Erdman SE, Alm EJ: Host lifestyle affects human microbiota on daily timescales. Genome Biology 2014, 15:R89-R89.

35. Brown RL, Clarke TB: The regulation of host defences to infection by the microbiota. Immunology 2017, 150:1-6.

36. Bach J-F: The hygiene hypothesis in autoimmunity: the role of pathogens and commensals. Nature Reviews Immunology 2017.

37. Carmody RN, Gerber GK, Luevano JM, Gatti DM, Somes L, Svenson KL, Turnbaugh PJ: Diet Dominates Host Genotype in Shaping the Murine Gut Microbiota. Cell Host \& Microbe 2015, 17:72-84.

38. David LA, Maurice CF, Carmody RN, Gootenberg DB, Button JE, Wolfe BE, Ling AV, Devlin AS, Varma $Y$, Fischbach MA, et al.: Diet rapidly and reproducibly alters the human gut microbiome. Nature 2014, 505:559-563.

39. Shoaie S, Ghaffari P, Kovatcheva-Datchary P, Mardinoglu A, Sen P, Pujos-Guillot E, De Wouters T, Juste C, Rizkalla S, Chilloux J, et al.: Quantifying Diet-Induced Metabolic Changes of the Human Gut Microbiome. Cell Metabolism 2015, 22:320-331.

40. Bokulich NA, Chung J, Battaglia T, Henderson N, Jay M, Li H, Lieber AD, Wu F, PerezPerez GI, Chen Y, et al.: Antibiotics, birth mode, and diet shape microbiome maturation during early life. Science Translational Medicine 2016, 8:1-14. 
41. Zeevi D, Korem T, Zmora N, Israeli D, Rothschild D, Weinberger A, Ben-Yacov O, Lador D, Avnit-Sagi T, Lotan-Pompan $\mathrm{M}$, et al.: Personalized Nutrition by Prediction of Glycemic Responses. Cell 2015, 163:1079-1095.

42. Rothschild D, Weissbrod O, Barkan E, Kurilshikov A, Korem T, Zeevi D, Costea PI, Godneva A, Kalka IN, Bar N, et al.: Environment dominates over host genetics in shaping human gut microbiota. Nature 2018, 555:210-215.

43. Wu H, Esteve E, Tremaroli V, Khan MT, Caesar R, Mannerås-Holm L, Ståhlman M, Olsson $L M$, Serino $M$, Planas-Fèlix $M$, et al.: Metformin alters the gut microbiome of individuals with treatment-naive type 2 diabetes, contributing to the therapeutic effects of the drug. Nature Medicine 2017, 23:850-858.

44. Cabreiro F, Au C, Leung K-Y, Vergara-Irigaray N, Cochemé HM, Noori T, Weinkove D, Schuster E, Greene NDE, Gems D: Metformin Retards Aging in C. elegans by Altering Microbial Folate and Methionine Metabolism. Cell 2013, 153:228-239.

45. Wallace BD, Wang H, Lane KT, Scott JE, Orans J, Koo JS, Venkatesh M, Jobin C, Yeh LA, Mani S, et al.: Alleviating cancer drug toxicity by inhibiting a bacterial enzyme. Science 2010, 330:831-835.

46. Scott TA, Quintaneiro LM, Norvaisas P, Lui PP, Wilson MP, Leung K-Y, HerreraDominguez L, Sudiwala S, Pessia A, Clayton PT, et al.: Host-Microbe Co-metabolism Dictates Cancer Drug Efficacy in C. elegans. Cell 2017, 169:1-15.

47. García-González AP, Ritter AD, Shrestha S, Andersen EC, Yilmaz LS, Walhout AJM: Bacterial Metabolism Affects the C. elegans Response to Cancer Chemotherapeutics. Cell 2017, 169:431-441.e438.

48. Yu TC, Guo F, Yu Y, Sun T, Ma D, Han J, Qian Y, Kryczek I, Sun D, Nagarsheth N, et al.: Fusobacterium nucleatum Promotes Chemoresistance to Colorectal Cancer by Modulating Autophagy. Cell 2017, 170:548-563.

49. Schroeder BO, Bäckhed F: Signals from the gut microbiota to distant organs in physiology and disease. Nature Medicine 2016, 22:1079-1089.

50. Zhu W, Winter MG, Byndloss MX, Spiga L, Duerkop BA, Hughes ER, Büttner L, De Lima Romão $\mathrm{E}$, Behrendt $\mathrm{CL}$, Lopez $\mathrm{CA}$, et al.: Precision editing of the gut microbiota ameliorates colitis. Nature 2018, 553:208-211.

51. Zou J, Chassaing B, Singh V, Pellizzon M, Ricci M, Fythe MD, Kumar MV, Gewirtz AT: Fiber-Mediated Nourishment of Gut Microbiota Protects against Diet-Induced Obesity by Restoring IL-22-Mediated Colonic Health. Cell Host \& Microbe 2018, 23:41-53.

52. Koeth RA, Wang Z, Levison BS, Buffa JA, Org E, Sheehy BT, Britt EB, Fu X, Wu Y, Li L, et al.: Intestinal microbiota metabolism of I-carnitine, a nutrient in red meat, promotes atherosclerosis. Nature Medicine 2013, 19:576-585.

53. Sonowal R, Swimm A, Sahoo A, Luo L, Matsunaga Y, Wu Z, Bhingarde JA, Ejzak EA, Ranawade $\mathrm{A}$, Qadota $\mathrm{H}$, et al.: Indoles from commensal bacteria extend healthspan. Proceedings of the National Academy of Sciences 2017, 114:E7506-E7515.

54. Devlin AS, Marcobal A, Dodd D, Nayfach S, Plummer N, Meyer T, Pollard KS, Sonnenburg JL, Fischbach MA: Modulation of a Circulating Uremic Solute via Rational Genetic Manipulation of the Gut Microbiota. Cell Host and Microbe 2016, 20:709-715.

55. Stappenbeck TS, Virgin HW: Accounting for reciprocal host-microbiome interactions in experimental science. Nature 2016, 534:191-199. 
56. Germerodt S, Bohl K, Lück A, Pande S, Schröter A, Kaleta C, Schuster S, Kost C: Pervasive Selection for Cooperative Cross-Feeding in Bacterial Communities. PLOS Computational Biology 2016, 12:1-21.

57. Thiele I, Clancy CM, Heinken A, Fleming RMT: Quantitative systems pharmacology and the personalized drug-microbiota-diet axis. Current Opinion in Systems Biology 2017, 4:43-52.

58. Kritikos G, Banzhaf M, Herrera-Dominguez L, Koumoutsi A, Wartel M, Zietek M, Typas A: A tool named Iris for versatile high-throughput phenotyping in microorganisms. Nature Microbiology 2017, 2:17014-17014.

59. Tramontano $M$, Andrejev S, Pruteanu $M$, Klünemann $M$ : Nutritional preferences of human gut bacteria reveal their metabolic idiosyncrasies. Nature Microbiology 2018, 3:4-6.

60. Bauer E, Zimmermann J, Baldini F, Thiele I, Kaleta C: BacArena: Individual-based metabolic modeling of heterogeneous microbes in complex communities. PLOS Computational Biology 2017, 13:e1005544-e1005544.

61. Esch EW, Bahinski A, Huh D: Organs-on-chips at the frontiers of drug discovery. Nature Reviews Drug Discovery 2015, 14:248-260.

62. Dirksen P, Marsh SA, Braker I, Heitland N, Wagner S, Nakad R, Mader S, Petersen C, Kowallik $\mathrm{V}$, Rosenstiel $\mathrm{P}$, et al.: The native microbiome of the nematode Caenorhabditis elegans: Gateway to a new host-microbiome model. BMC Biology 2016, 14:1-16.

63. Samuel BS, Rowedder H, Braendle C, Félix M-A, Ruvkun G: Caenorhabditis elegans responses to bacteria from its natural habitats. Proceedings of the National Academy of Sciences 2016, 113:E3941-E3949.

64. Cabreiro F, Gems D: Worms need microbes too: microbiota, health and aging in Caenorhabditis elegans. EMBO Molecular Medicine 2013, 5:1300-1310.

65. Piper MDW, Blanc E, Leitão-Gonçalves R, Yang M, He X, Linford NJ, Hoddinott MP, Hopfen C, Soultoukis GA, Niemeyer C, et al.: A holidic medium for Drosophila melanogaster. Nature Methods 2014, 11:100-105.

66. Clark RI, Walker DW: Role of gut microbiota in aging-related health decline: insights from invertebrate models. Cellular and Molecular Life Sciences 2017, 75:1-9.

67. Leitão-Gonçalves R, Carvalho-Santos Z, Francisco AP, Fioreze GT, Anjos M, Baltazar C, Elias AP, Itskov PM, Piper MDW, Ribeiro C: Commensal bacteria and essential amino acids control food choice behavior and reproduction. PLOS Biology 2017, 15:e2000862-e2000862.

68. Storelli G, Strigini M, Grenier T, Bozonnet L, Schwarzer M, Daniel C, Matos R, Leulier F: Drosophila Perpetuates Nutritional Mutualism by Promoting the Fitness of Its Intestinal Symbiont Lactobacillus plantarum. Cell Metabolism 2018, 27:362377.e368.

69. Stephens WZ, Wiles TJ, Martinez ES, Jemielita M, Burns AR, Parthasarathy R, Bohannan BJM, Guillemin K: Identification of population bottlenecks and colonization factors during assembly of bacterial communities within the zebrafish intestine. mBio 2015, 6:1-11.

70. Smith P, Willemsen D, Popkes M, Metge F, Gandiwa E, Reichard M, Valenzano DR: Regulation of life span by the gut microbiota in the short-lived African turquoise killifish. elife 2017, 6:1-26. 
71. Dodd D, Spitzer MH, Van Treuren W, Merrill BD, Hryckowian AJ, Higginbottom SK, Le A, Cowan TM, Nolan GP, Fischbach MA, et al.: A gut bacterial pathway metabolizes aromatic amino acids into nine circulating metabolites. Nature 2017, 551:648-652.

72. Biggs MB, Medlock GL, Moutinho TJ, Lees HJ, Swann JR, Kolling GL, Papin JA: Systemslevel metabolism of the Altered Schaedler Flora, a complete gut microbiota. The ISME Journal 2016, 11:1-13.

73. Turnbaugh PJ, Ridaura VK, Faith JJ, Rey FE, Knight R, Gordon JI: The effect of Diet on the Human Gut Microbiom: A Metagenomic Analysis in Humanized Gnotobiotic Mice. Science translational medicine 2009, 1:1-19.

74. Wang M, Donovan SM: Human microbiota-associated swine: Current progress and future opportunities. ILAR Journal 2015, 56:63-73.

75. Watson E, Macneil LT, Ritter AD, Yilmaz LS, Rosebrock AP, Caudy AA, Walhout AJM: Interspecies systems biology uncovers metabolites affecting $C$. elegans gene expression and life history traits. Cell 2014, 156:759-770.

76. Baba T, Ara T, Hasegawa M, Takai Y, Okumura Y, Baba M, Datsenko Ka, Tomita M, Wanner BL, Mori H: Construction of Escherichia coli K-12 in-frame, single-gene knockout mutants: the Keio collection. Molecular Systems Biology 2006, 2:1-11.

77. Han B, Sivaramakrishnan P, Lin CCJ, Neve IAA, He J, Tay LWR, Sowa JN, Sizovs A, Du G, Wang J, et al.: Microbial Genetic Composition Tunes Host Longevity. Cell 2017, 169:1249-1262.

78. Virk B, Jia J, Maynard CA, Raimundo A, Lefebvre J, Richards SA, Chetina N, Liang Y, Helliwell N, Cipinska M, et al.: Folate Acts in E. coli to Accelerate C. elegans Aging Independently of Bacterial Biosynthesis. Cell Reports 2016, 14:1611-1620.

79. Rual J-F, Ceron J, Koreth J, Hao T, Nicot A-S, Hirozane-Kishikawa T, Vandenhaute J, Orkin SH, Hill DE, Heuvel Svd, et al.: Toward Improving Caenorhabditis elegans Phenome Mapping With an ORFeome-Based RNAi Library. Genome Research 2004, 14:21622168.

80. Thompson O, Edgley M, Strasbourger P, Flibotte S, Ewing B, Adair R, Au V, Chaudhry I, Fernando $\mathrm{L}$, Hutter $\mathrm{H}$, et al.: The million mutation project: a new approach to genetics in Caenorhabditis elegans. Genome research 2013, 23:1749-1762.

81. Yan A, Culp E, Perry J, Lau JT, MacNeil LT, Surette MG, Wright GD: Transformation of the Anticancer Drug Doxorubicin in the Human Gut Microbiome. ACS Infectious Diseases 2017, 4:68-76.

82. Zaslaver A, Bren A, Ronen M, Itzkovitz S, Kikoin I, Shavit S, Liebermeister W, Surette MG, Alon $\mathrm{U}$ : A comprehensive library of fluorescent transcriptional reporters for Escherichia coli. Nature Methods 2006, 3:623-628.

83. Aurich MK, Fleming RMT, Thiele I: MetaboTools: A Comprehensive Toolbox for Analysis of Genome-Scale Metabolic Models. Frontiers in Physiology 2016, 7.

84. Magnúsdóttir S, Heinken A, Kutt L, Ravcheev DA, Bauer E, Noronha A, Greenhalgh K, Jäger C, Baginska J, Wilmes $P$, et al.: Generation of genome-scale metabolic reconstructions for $\mathbf{7 7 3}$ members of the human gut microbiota. Nature Biotechnology 2016, 35:81-89.

85. Hastings J, Mains A, Artal-Sanz M, Bergmann S, Braeckman BP, Bundy J, Cabreiro F, Dobson P, Ebert P, Hattwell J, et al.: WormJam: A consensus C. elegans Metabolic Reconstruction and Metabolomics Community and Workshop Series. Worm 2017, 6:e1373939. 
a

\section{Holobiont Environment}

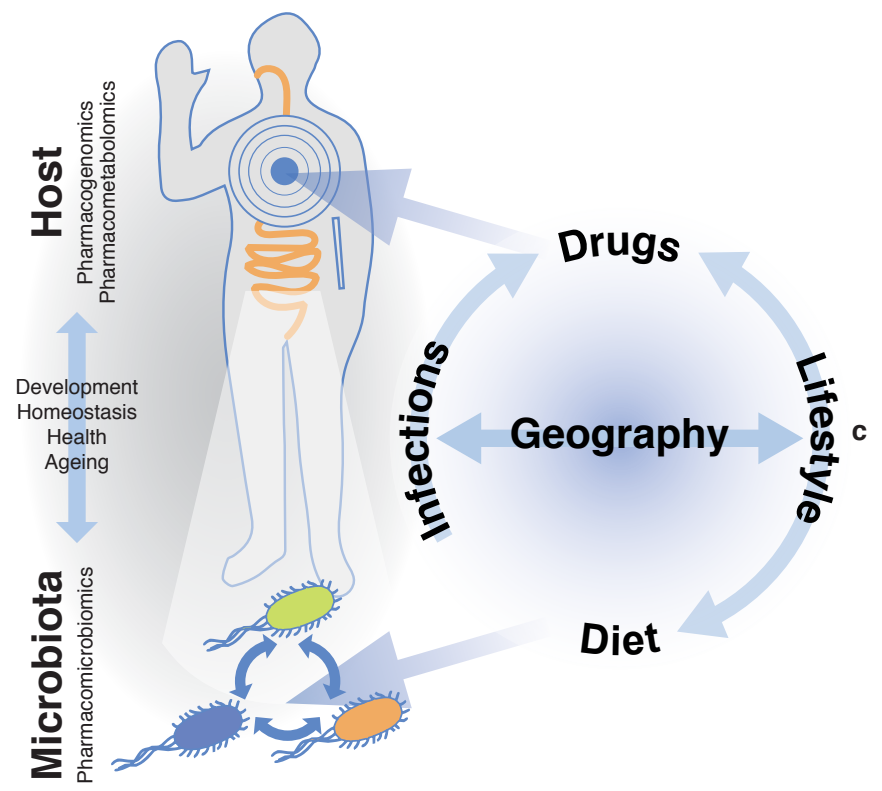

Microbial ecology b

\section{Xenobiotics \\ (Diet \& Drugs)}

Effect on microbe

Nutrient//

Positive

Dietary Carbohydrates

fibre

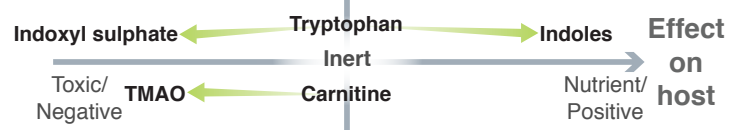

Antibiotics

Toxic/

Negative

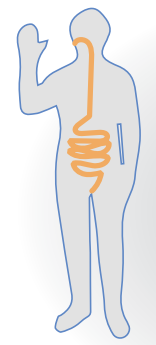

Metabonome

$\sim 30,000$ genes

Static
Metagenome $\sim 3,000,000$ genes Moldable 


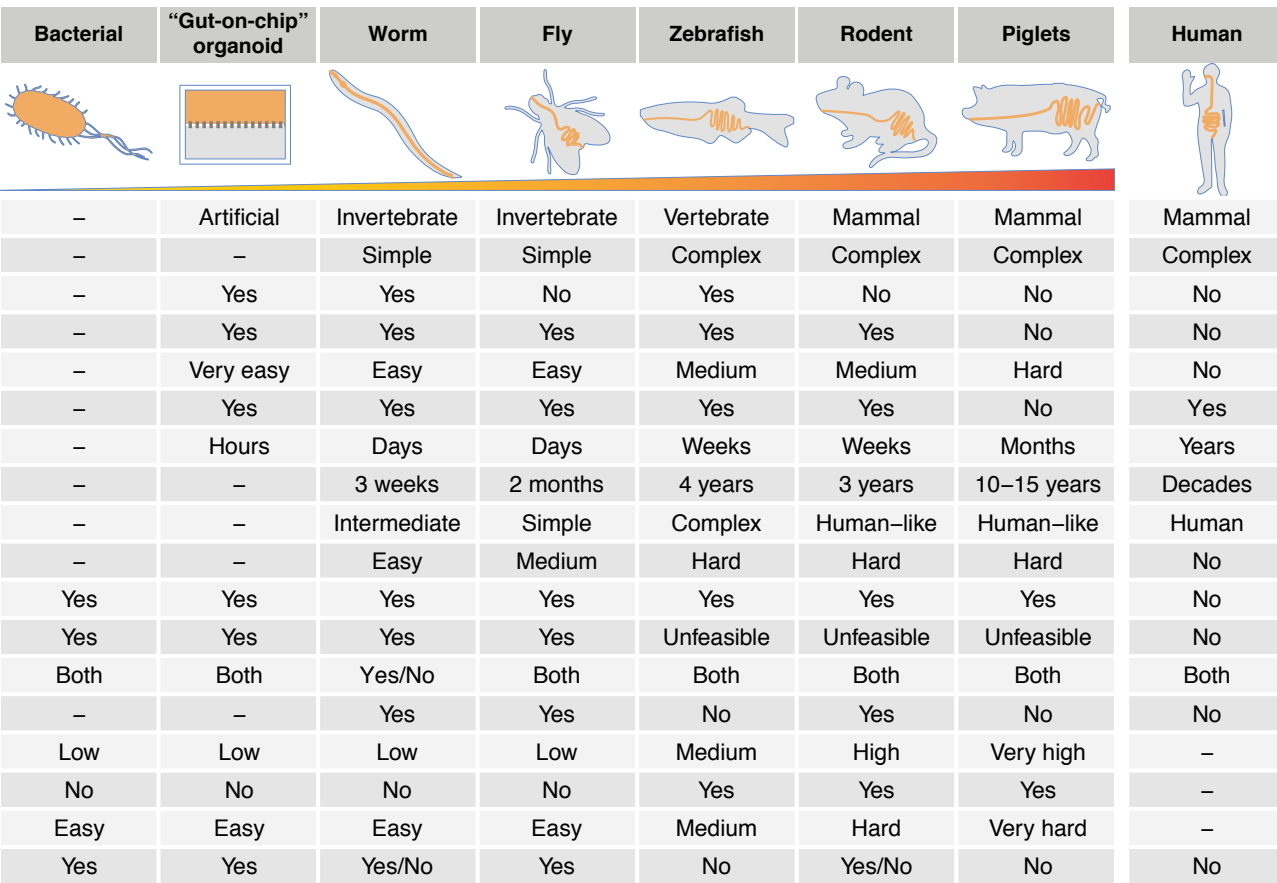

b Experimental C. elegans holobiont model



c Metabolic C. elegans holobiont model Xenobiotics

(Diet \& Drugs)

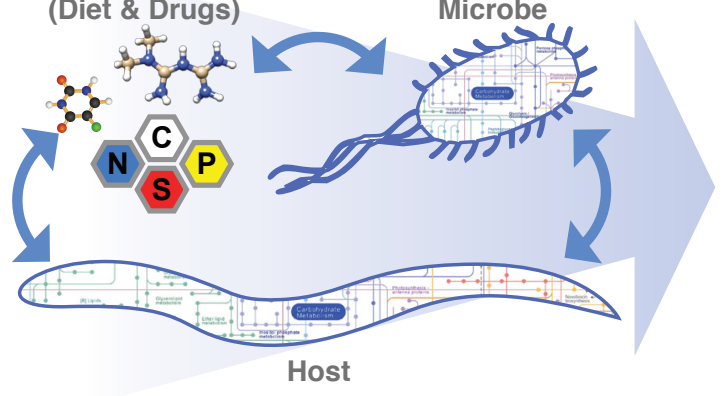

Metabolic human holobiont model

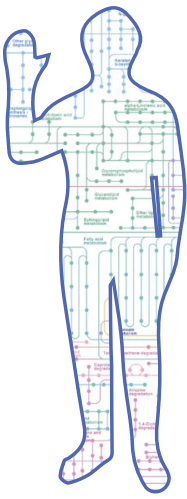

